\title{
The effect of the anomalous Wtb interactions on spin correla- tions in t-channel single top quark production with subsequent decay
}

\author{
Eduard Boos ${ }^{1,2, \star}$ and Viacheslav Bunichev ${ }^{1, \star \star}$ \\ ${ }^{1}$ Skobeltsyn Institute of Nuclear Physics, Lomonosov Moscow State University Leninskie Gory, 119991, \\ Moscow, Russia \\ ${ }^{2}$ Faculty of Physics, Lomonosov Moscow State University, Leninskie Gory, 119991, Moscow, Russia
}

\begin{abstract}
Spin correlations in the t-channel single top quark production and its subsequent decay are investigated for the case of contributions involving anomalous Wtb couplings. It is shown that shapes of certain multidimensional kinematic distributions of final state particles are significantly different for the contributions proportional to different combinations of the anomalous couplings. Observed properties are confirmed by two methods of computations either from the obtained symbolic expression for the differential width of polarized three-particle top quark decay or by means of the CompHEP program for the complete process involving the t-channel single top.
\end{abstract}

\section{Introduction}

With the Higgs boson discovery at the LHC the Standard Model (SM) is completed in a sense that all predicted particles are found and all the interactions between particles are fixed. However, most likely, the SM is a kind of effective field theory working (and working amazingly well) at the energy range determined by the electroweak (EW) energy scale. The top quark being the heaviest found fundamental particle with closest to the EW scale mass is a promising place to search for possible deviations from the SM (see, recent reviews on top quark [1-5]. In particular such deviations may be related to a presence of the top quark anomalous couplings which are usually parametrizes in terms of a number of the gauge invariant dimension 6 operators [6-10]

$$
\begin{array}{ll}
O_{\phi q}^{(3,3+3)}=\frac{i}{2}\left[\phi^{\dagger}\left(\tau^{I} D_{\mu}-\overleftarrow{D}_{\mu} \tau^{I}\right) \phi\right]\left(\bar{q}_{L 3} \gamma^{\mu} \tau^{I} q_{L 3}\right), & O_{\phi \phi}^{33}=i\left(\tilde{\phi}^{\dagger} D_{\mu} \phi\right)\left(\bar{t}_{R} \gamma^{\mu} b_{R}\right) \\
O_{d W}^{33}=\left(\bar{q}_{L 3} \sigma^{\mu v} \tau^{I} b_{R}\right) \phi W_{\mu \nu}^{I}, & O_{u W}^{33}=\left(\bar{q}_{L 3} \sigma^{\mu v} \tau^{I} t_{R}\right) \tilde{\phi} W_{\mu \nu}^{I}
\end{array}
$$

These operators lead to the effective Lagrangian [11] allowed by the Lorentz invariance parametrizing the anomalous terms in Wtb vertex

\footnotetext{
^e-mail: boos@theory.sinp.msu.ru

${ }^{\star}$ e-mail: bunichev@ theory.sinp.msu.ru
} 


$$
\begin{aligned}
\mathcal{L}= & \frac{g}{\sqrt{2}} \bar{b} \gamma^{\mu}\left(f_{L V} P_{L}+f_{R V} P_{R}\right) t W_{\mu}^{-} \\
& +\frac{g}{\sqrt{2}} \bar{b} \frac{\sigma^{\mu \nu}}{2 M_{W}}\left(f_{L T} P_{L}+f_{R T} P_{R}\right) t W_{\mu \nu}^{-}+\text {h.c. }
\end{aligned}
$$

where $M_{W}$ is the W-boson mass, $P_{L, R}=\left(1 \mp \gamma_{5}\right) / 2$ is the left(right)-handed projection operator, $W_{\mu \nu}^{-}=\partial_{\mu} W_{\nu}^{-}-\partial_{\nu} W_{\mu}^{-}, \sigma^{\mu \nu}=i / 2\left[\gamma_{\mu}, \gamma_{\nu}\right], g$ is the weak isospin gauge coupling, parameters $f_{L V(T)}$ and $f_{R V(T)}$ are the dimensionless coefficients that parametrize strengths of the left-vector (tensor) and the right-vector (tensor) structures in the Lagrangian.

The couplings in the Lagrangian (2) are related in the following way to constants $C_{\phi q}^{(3,3+3)}, C_{\phi \phi}^{33}, C_{d W}^{33}, C_{u W}^{33}$ in front of the gauge-invariant dimension six operators : $f_{L V}=V_{t b}+$ $C_{\phi q}^{(3,3+3)} \frac{v^{2}}{\Lambda^{2}}, \quad f_{R V}=\frac{1}{2} C_{\phi \phi}^{33} \frac{v^{2}}{\Lambda^{2}}, \quad f_{L T}=\sqrt{2} C_{d W}^{33} \frac{v^{2}}{\Lambda^{2}}, f_{R T}=\sqrt{2} C_{u W}^{33} \frac{v^{2}}{\Lambda^{2}}$.

If one assumes that naturally the constants in front of the operators are of the order of unity, the natural values for the anomalous couplings are of the order of $\frac{v^{2}}{\Lambda^{2}}$ and therefore rather small. This is confirmed by the recent most stringent experimental limits [12].

In the SM all fermions interact through the left-handed currents and all constants are equal to zero, except $f_{L V}=V_{t b}$ (CKM-matrix element). Note that the anomalous coupling parameters could be complex in the most general case. However here we consider only real anomalous couplings for simplicity.

In this short paper we discuss a simple idea allowing to improve further the limits on anomalous couplings. The idea is based on the top quark spin correlation properties in the t- and s-channel single top production with its subsequent decay. Spin correlations in presence of anomalous couplings have been studied previously in $[9,13-15]$

As well known in the SM, the positively charged lepton from the top quark decay in its rest frame tends to follow the top quark spin direction [16, 17]. In the t- and s-channel single top production the direction of the top quark spin in the top rest frame is highly correlated with the d-quark momentum for the t-channel (outgoing light jet) and anti d-quark momentum (incoming parton corresponding to the beam axis) for the s-channel $[18,19]$. One can understand this very simply by considering the single top production as a decay back in time [20]. The direction of preferred spin configuration in the single top production in presence of anomalous couplings is changed insignificantly comparing to SM due to smallness of the anomalous couplings. So one can chose the direction related to mentioned d-quark momentum as a top spin quantization axis and to make sure that this will be a preferred spin direction of top quark in its rest frame similar to SM. After production the highly polarized top quark decays (Fig. 1), and one can use properties of such a polarized decay.

\section{Top decay}

Symbolic computation gives the following expression for the differential decay width of the top quark in its rest frame. We integrate the matrix element squared of the polarized t-quark decay over the neutrino and b-quark momenta using the narrow width approximation for the $\mathrm{W}$-boson decay and neglecting the b-quark mass in comparison to the top quark and W-boson masses: 


$$
\begin{aligned}
& \frac{d \Gamma_{t \rightarrow b v e^{+}}}{d E_{e} \cdot d \cos \theta \cdot d \phi}=\frac{g^{4}}{256 \cdot \pi^{3} \cdot \Gamma_{W} \cdot m_{W}} \cdot[ \\
& +\left(f_{L V}^{2} \cdot\left(E_{\text {max }}-E_{e}\right)+f_{L T}^{2} \cdot\left(E_{e}-E_{\text {min }}\right)\right) \cdot E_{e} \cdot(1+\cos \theta) \\
& +\left(f_{R T}^{2} \cdot\left(E_{\text {max }}-E_{e}\right)+f_{R V}^{2} \cdot\left(E_{e}-E_{\text {min }}\right)\right) \times \\
& \quad\left(E_{\text {min }}+E_{\text {max }}-E_{e}+m_{W} / E_{e} \cdot c_{e} \cdot \sin \theta \cos \phi+\left(m_{W}^{2} /\left(2 E_{e}\right)+E_{e}-E_{\text {min }}-E_{\text {max }}\right) \cdot \cos \theta\right) \\
& \left.-2 \cdot\left(f_{L V} \cdot f_{R T} \cdot\left(E_{\text {max }}-E_{e}\right)+f_{L T} \cdot f_{R V} \cdot\left(E_{e}-E_{\text {min }}\right)\right) \cdot\left(c_{e} \cdot \sin \theta \cos \phi+m_{W} / 2 \cdot(1+\cos \theta)\right)\right]
\end{aligned}
$$

where:

$$
c_{e}=\sqrt{\left(E_{\max }-E_{e}\right) \cdot\left(E_{e}-E_{\min }\right)}, \quad E_{\max }=m_{t} / 2, \quad E_{\min }=m_{W}^{2} /\left(2 m_{t}\right)
$$

Here the angle $\theta$ is the angle between the charged lepton momentum and the direction of the top quark spin quantization axis, the angle $\phi$ is the angle in the plane perpendicular to the lepton momenta counted from the decay plain formed by the top quark decay products as shown in Fig. $2, E_{e}$ is the energy of the charged lepton.

The expression (3) with real anomalous couplings contains of 4 terms corresponding to different combinations the coupling products. Each coupling combination is multiplied by an individual polynomial kinematic function depending on $\theta, \phi$ and $E_{e}$. Therefore such multidimensional distribution shape (multidimensional surface) can be used to separate better contributions from different anomalous coupling combinations.

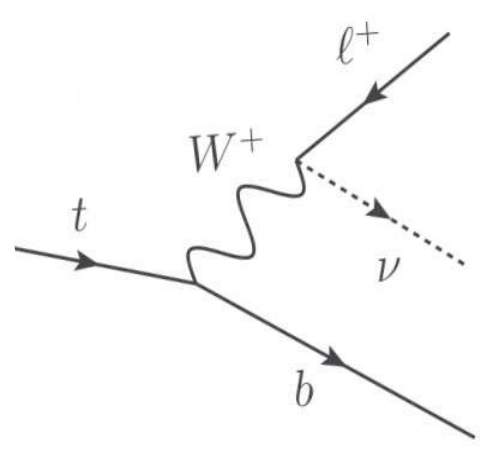

Figure 1. Top quark leptonic decay process.

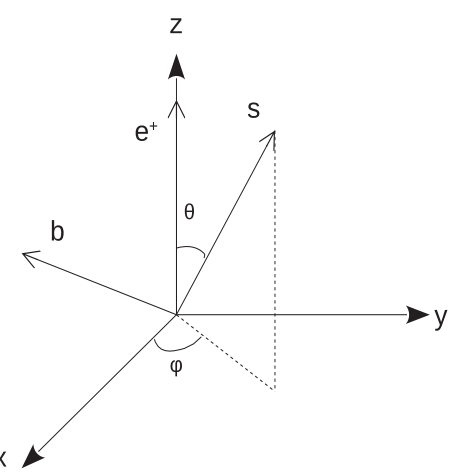

Figure 2. Top quark spin quantization axis.

The first term in the expression [16, 17] is the well-known SM like contribution corresponding to $100 \%$ spin correlation behavior $(1+\cos \theta)$ with the lepton energy distribution having a maximum at $m_{t} / 4$. The interference term between the left-vector and the right-tensor terms coincides exactly with the expression presented in [9]. 


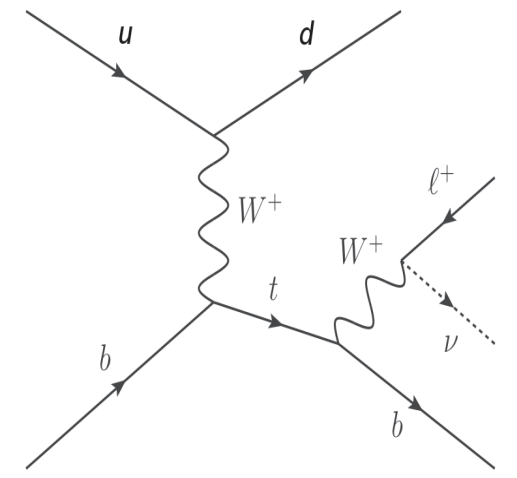

Figure 3. t-channel single top quark production and its leptonic decay processes.

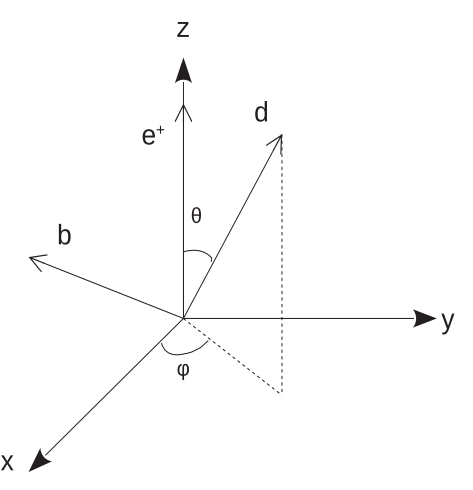

Figure 4. Top quark spin quantization axis in the top rest frame is chosen as the direction of d-quark momentum for the t-channel (outgoing light jet).

\section{Numerical illustration}

Numerical illustration we present for few cases performing computations by two different methods in each case. First, we take the differential width (3) and plot the corresponding surface. Second, we perform computations and Monte-Carlo event generation for the $2 \rightarrow 4$ process corresponding to the dominating t-channel single production with the top quark 3 body decay $u b \rightarrow l^{+} v_{l} b d$ (Fig. 4) using the CompHEP code version [21]. For the latter computation we show the same multidimensional distributions in the top rest frame for proton-proton collisions at $13 \mathrm{TeV}$ energy taking all the angles with respect to the d-quark momentum. To make deviations from the SM more pronounce we subtract the SM contribution and we integrate in both ways of computations over the variable $\phi$ in the some range (for definiteness the range was taken from $-\pi / 3$ to $+\pi / 3$ ). We take values for the anomalous couplings from in correspondence with the recent experimental upper limits [12].

1. Non-zero left vector coupling $f_{L V}\left(\left|f_{L V}-V_{t b}\right|=0.1\right)$, other anomalous couplings are equal to zero. Contributing terms of the (3) are proportional to $\left(f_{L V}-V_{t b}\right) \cdot\left(f_{L V}-V_{t b}\right)$ and $\left(f_{L V}-V_{t b}\right) \cdot V_{t b}$. Fig. 5 shows distribution $\left.\frac{1}{\Gamma-\Gamma_{S M}} d\left(\Gamma-\Gamma_{S M}\right) /\left(d E_{e} \cdot d \cos \theta\right)\right)$. Because of the dominating interference term of the left vector anomalous coupling $\left(f_{L V}-V_{t b}\right)$ with the SM $V_{t b}$ coupling the maximum of the distribution is located as expected at $\cos \theta=1$ and $E_{e}=m_{t} / 4 \approx 43 \mathrm{GeV}$. The distribution almost linearly drops to 0 with decreasing $\cos \theta$. The distribution from the CompHEP simulation is shown in Fig. 6. Good agreement is observed.

2. Non-zero right vector coupling $f_{R V}=0.16$, other anomalous couplings are equal to zero. The contributing term of the (3) is proportional to $\left|f_{R V}\right|^{2}$. Two dimensional distribution shown in Fig. 7 agrees well with the CompHEP computations given in Fig. 8. The maximum of is located now at the values $\cos \theta \approx 0.3$ and $E_{e} \approx 60$. One can see that the shape of the distribution in this case is significantly different from that in case 1 .

3. Non-zero left tensor coupling $f_{L T}=0.057$, other anomalous couplings are equal to zero. The contributing term of the (3) is proportional to $\left|f_{L T}\right|^{2}$, and the distribution in Fig. 9 is very similar to that from the CompHEP computation (Fig. 10). The maximum is located at $\cos \theta=1$ and $E_{e}=E_{\max } \approx$ $86.25 \mathrm{GeV}$. With decreasing $\cos \theta$ and $E_{e}$ the distribution quickly goes to 0 .

4. Non-zero right tensor coupling $f_{R T}=0.048$, other anomalous couplings are equal to zero. The contributing terms of the (3) are proportional to the squared $\left|f_{R T}\right|^{2}$ and to the interference $R e f_{R T} \cdot V_{t b}$ 


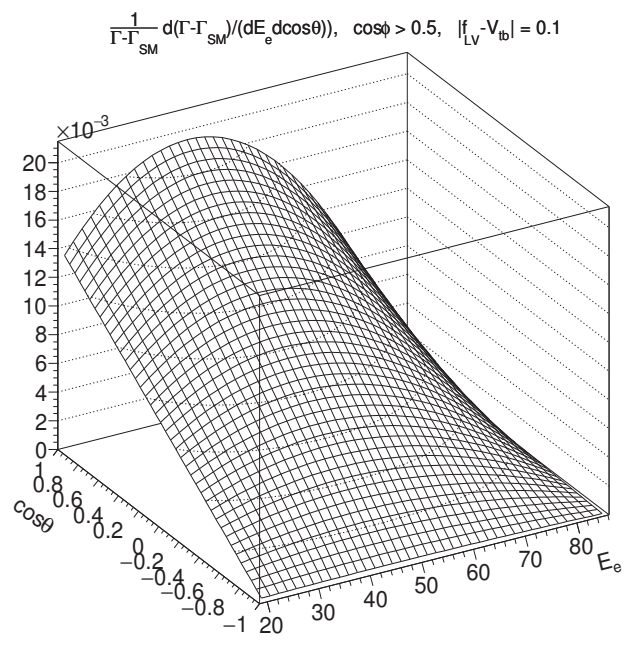

Figure 5. Top quark decay, $\left|f_{L V}-V_{t b}\right|=0.1$.

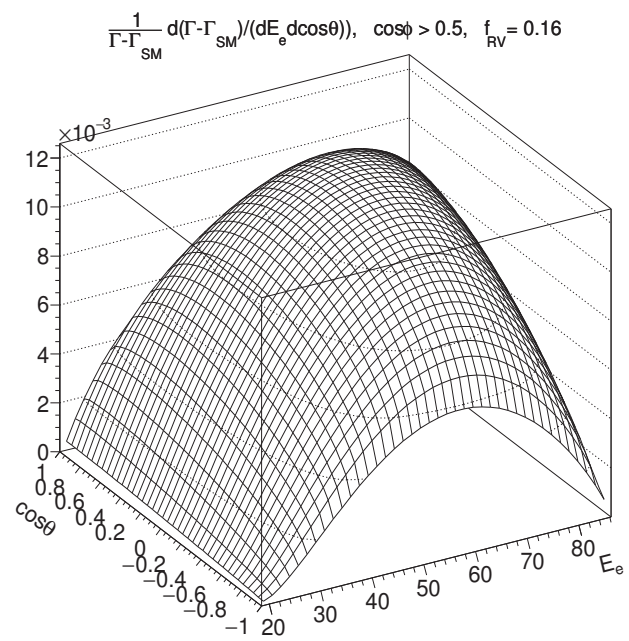

Figure 7. Top quark decay, $f_{R V}=0.16$.

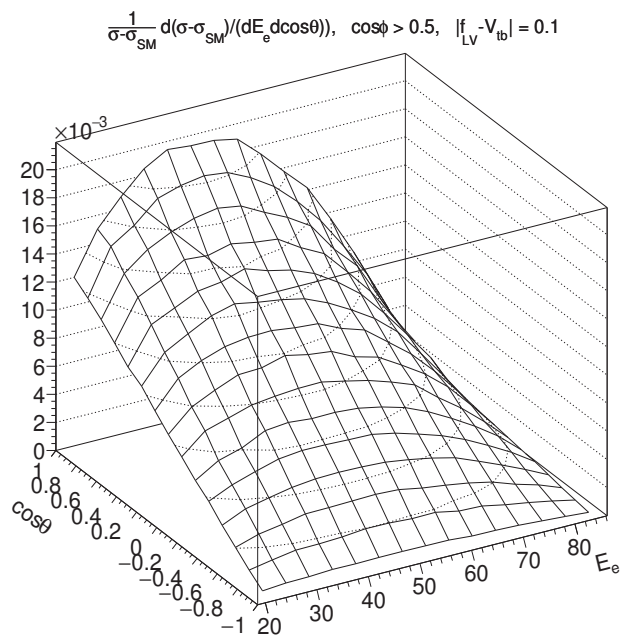

Figure 6. Top quark production and decay, $\left|f_{L V}-V_{t b}\right|=$ 0.1 .

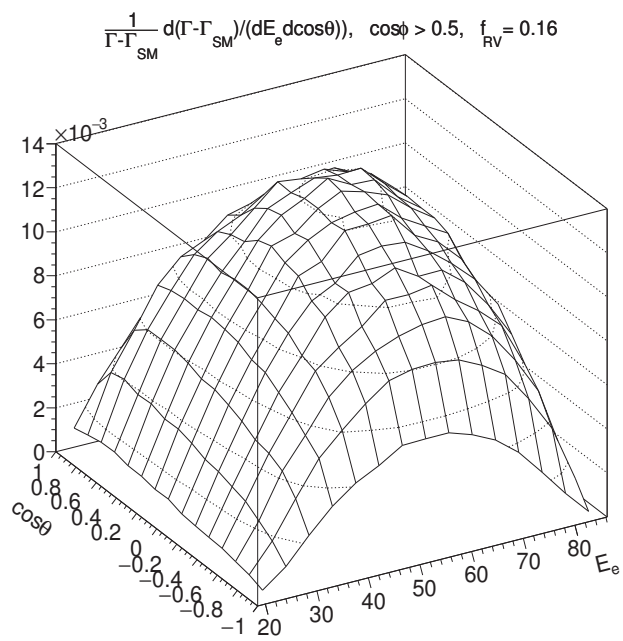

Figure 8. Top quark production and decay $f_{R V}=0.16$.

parts. Once more the complete agreement between computations using the formula 3 for the width (Fig. 11) and by means of the CompHEP (Fig. 12). With decreasing $\cos \theta$ and increasing $E_{e}$ the distribution shape almost linearly drops to 0 .

One can clearly see that the shapes of distributions proportional to different anomalous couplings are significantly different. 


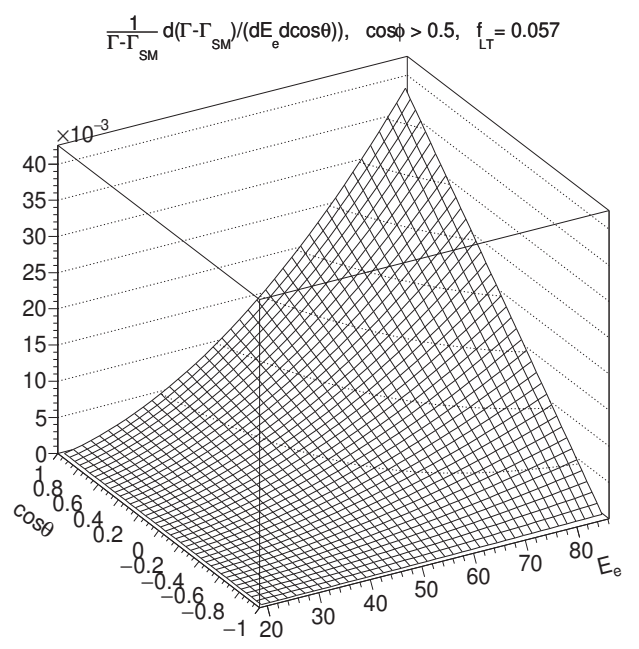

Figure 9. Top quark decay, $f_{L T}=0.057$.

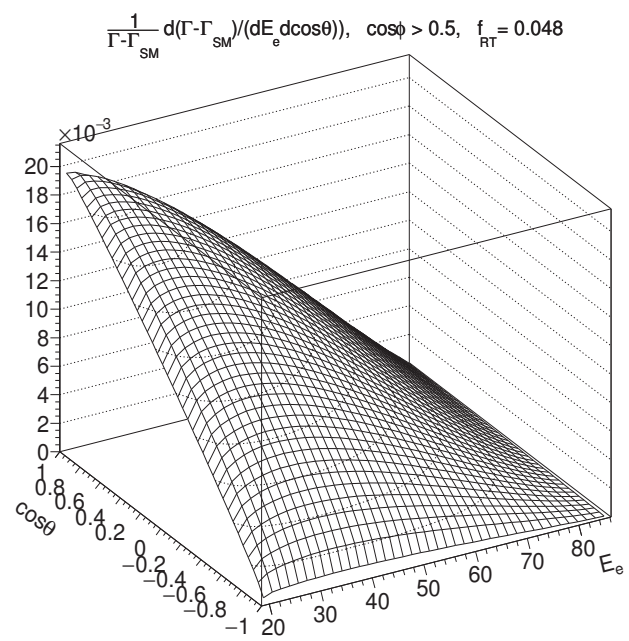

Figure 11. Top quark decay, $f_{R T}=0.048$.

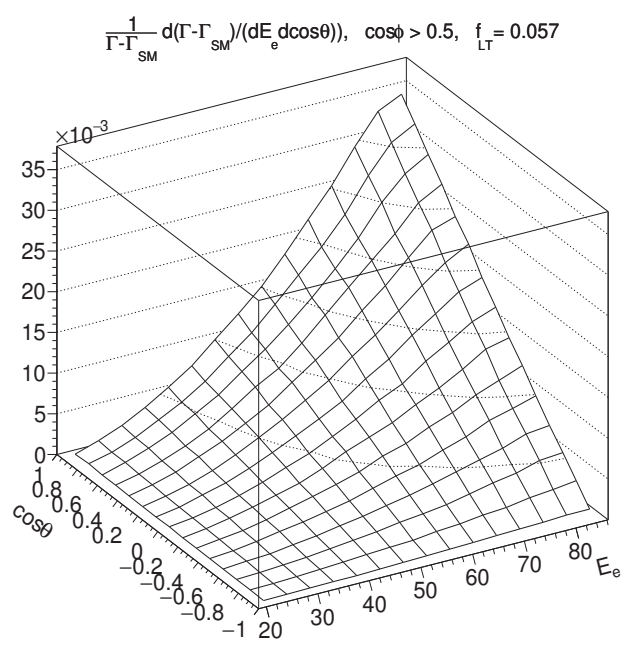

Figure 10. Top quark production and decay $f_{L T}=$ 0.057 .

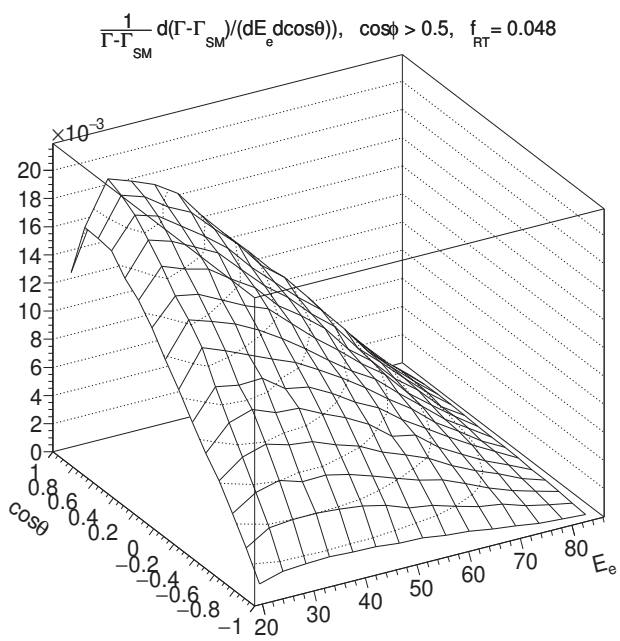

Figure 12. Top quark production and decay $f_{R T}=$ 0.048 .

\section{Conclusions}

We obtained the analytic expression for the differential width for the three-particle decay of polarized $\mathrm{t}$-quark in the its rest frame as a function of orientation angles of the top quark spin quantization axis and charged lepton energy. The expression is presented for the case of real vector and tensor anomalous Wtb couplings. The parts of this expression containing the SM contribution and its interference with anomalous contributions are in complete agreement with published results. 
Using this formula for the differential width we constructed two-dimensional distributions on the angle between the direction of the charged lepton momentum and the top quark spin quantization axis and on the p energy of the charged lepton. We plot such a distributions separately for each of the contribution proportional to anomalous couplings and shown significant differences in shapes of the distributions. These differences can be extracted form the final products of the t-channel single top production process with the top decay taking into account that due to smallness of the anomalous couplings the top is produced highly polarized in the direction of the d-quark produced giving correspondingly the best choice for top quark spin quantization axis.

We have confirmed the forms of the distributions by numerical computation of the complete process involving the t-channel top quark production and its decay and generation of the corresponding Monte-Carlo events using the Comp Hep package. From the generated events we constructed the same two-dimensional differential distributions for each anomalous contribution. It was shown that these distributions are in a very good agreement with the distributions predicted by the derived analytic formula. The differences in shapes of the distributions allows one to get limits on anomalous couplings in addition to the limits already extracted by other methods. Details of an application of such a method will be presented in a separate study.

\section{Acknowledgments}

The work was supported by grant 16-12-10280 of Russian Science Foundation. The authors are grateful to L. Dudko for useful discussions and critical remarks.

\section{References}

[1] U. Husemann, “Top-Quark Physics: Status and Prospects,” Prog. Part. Nucl. Phys. 95 (2017) 48

[2] M. Cristinziani and M. Mulders, "Top-quark physics at the Large Hadron Collider," J. Phys. G 44 (2017) no.6, 063001

[3] E. Boos, O. Brandt, D. Denisov, S. Denisov and P. Grannis, "The top quark (20 years after its discovery)," Phys. Usp. 58, no. 12, 1133 (2015) [Usp. Fiz. Nauk 185, no. 12, 1241 (2015)]

[4] C. E. Gerber and C. Vellidis, "Review of Tevatron results: Top quark physics," Int. J. Mod. Phys. A 30, no. 06, 1541005 (2015).

[5] F. Déliot, N. Hadley, S. Parke and T. Schwarz, "Properties of the Top Quark," Ann. Rev. Nucl. Part. Sci. 64, 363 (2014).

[6] K. Whisnant, J. M. Yang, B. L. Young and X. Zhang, "Dimension-six CP conserving operators of the third family quarks and their effects on collider observables," Phys. Rev. D 56, 467 (1997)

[7] E. Boos, M. Dubinin, M. Sachwitz and H. J. Schreiber, "Probe of the W t b coupling in t anti-t pair production at linear colliders," Eur. Phys. J. C 16 (2000) 269

[8] J. A. Aguilar-Saavedra, "Single top quark production at LHC with anomalous Wtb couplings," Nucl. Phys. B 804, 160 (2008)

[9] C. Zhang and S. Willenbrock, "Effective-Field-Theory Approach to Top-Quark Production and Decay," Phys. Rev. D 83, 034006 (2011)

[10] J. L. Birman, F. Déliot, M. C. N. Fiolhais, A. Onofre and C. M. Pease, "New limits on anomalous contributions to the Wtb vertex," Phys. Rev. D 93, no. 11, 113021 (2016)

[11] G. L. Kane, G. A. Ladinsky and C. P. Yuan, "Using the Top Quark for Testing Standard Model Polarization and CP Predictions," Phys. Rev. D 45, 124 (1992). 
[12] V. Khachatryan et al. [CMS Collaboration], "Search for anomalous Wtb couplings and flavourchanging neutral currents in t-channel single top quark production in pp collisions at $\sqrt{s}=7$ and 8 TeV," JHEP 1702, 028 (2017)

[13] J. A. Aguilar-Saavedra and S. Amor Dos Santos, "New directions for top quark polarization in the $t$-channel process," Phys. Rev. D 89, no. 11, 114009 (2014)

[14] J. A. Aguilar-Saavedra, "Missing Top Properties,” arXiv:1411.5697 [hep-ph].

[15] J. A. Aguilar-Saavedra, C. Degrande and S. Khatibi, "Single top polarisation as a window to new physics," Phys. Lett. B 769 (2017) 498

[16] M. Jezabek and J. H. Kuhn, "V-A tests through leptons from polarized top quarks," Phys. Lett. B 329, 317 (1994)

[17] M. Jezabek, “Top quark physics,” Nucl. Phys. Proc. Suppl. 37B, no. 2, 197 (1994)

[18] G. Mahlon and S. J. Parke, "Improved spin basis for angular correlation studies in single top quark production at the Tevatron," Phys. Rev. D 55, 7249 (1997)

[19] G. Mahlon and S. J. Parke, "Single top quark production at the LHC: Understanding spin," Phys. Lett. B 476, 323 (2000)

[20] E. E. Boos and A. V. Sherstnev, "Spin effects in processes of single top quark production at hadron colliders," Phys. Lett. B 534, 97 (2002)

[21] E. Boos et al. [CompHEP Collaboration], "CompHEP 4.4: Automatic computations from Lagrangians to events,” Nucl. Instrum. Meth. A 534, 250 (2004) 